On the wurtzite to tetragonal phase transformation in $\mathrm{ZnO}$ nanowires

This content has been downloaded from IOPscience. Please scroll down to see the full text. 2017 Nanotechnology 28165705

(http://iopscience.iop.org/0957-4484/28/16/165705)

View the table of contents for this issue, or go to the journal homepage for more

Download details:

IP Address: 124.16.154.202

This content was downloaded on 15/08/2017 at 10:14

Please note that terms and conditions apply.

You may also be interested in:

Mechanical properties of monocrystalline and polycrystalline monolayer black phosphorus Pinqiang Cao, Jianyang Wu, Zhisen Zhang et al.

Orientation and size dependence of the elastic properties of zinc oxide nanobelts

A J Kulkarni, M Zhou and F J Ke

Effects of temperature and strain rate on the mechanical properties of hexagonal boron nitride nanosheets

Tongwei Han, Ying Luo and Chengyuan Wang

Anomalous surface states modify the size-dependent mechanical properties and fracture of silica nanowires

Chun Tang and Lilian P Dávila

Void initiation from interfacial debonding of spherical silicon particles inside a silicon-copper nanocomposite: a molecular dynamics study

Yi Cui and Zengtao Chen

Dynamic behaviour of silicon carbide nanowires under high and extreme strain rates: a molecular dynamics study

H Tsuzuki, J P Rino and P S Branicio

$\underline{\text { Tunable thermal response of } \mathrm{ZnO} \text { nanowires }}$

A J Kulkarni and M Zhou

Nanomechanical modeling of a (100)[001] crack in a single crystal bcc iron cantilever beam Jørn Skogsrud, Marie Jørum and Christian Thaulow 


\title{
On the wurtzite to tetragonal phase transformation in $\mathrm{ZnO}$ nanowires
}

\author{
Jun Wang ${ }^{1}$, Yaogen Shen ${ }^{2}$, Fan Song ${ }^{1}$, Fujiu $\mathrm{Ke}^{3}$, Xiaozhou Liao ${ }^{4}$ and \\ Chunsheng $\mathrm{Lu}^{5}$ \\ ${ }^{1}$ State Key Laboratory of Nonlinear Mechanics (LNM), Institute of Mechanics, Chinese Academy of \\ Sciences, Beijing 100190, People's Republic of China \\ ${ }^{2}$ Department of Mechanical and Biomedical Engineering, City University of Hong Kong, Kowloon, Hong \\ Kong, People's Republic of China \\ ${ }^{3}$ School of Physics and Nuclear Energy Engineering, Beihang University, Beijing 100191, People's \\ Republic of China \\ ${ }^{4}$ School of Aerospace, Mechanical and Mechatronic Engineering, University of Sydney, Sydney, NSW \\ 2006, Australia \\ ${ }^{5}$ Department of Mechanical Engineering, Curtin University, Perth, WA 6845, Australia \\ E-mail: wangjun@lnm.imech.ac.cn
}

Received 29 November 2016, revised 20 February 2017

Accepted for publication 8 March 2017

Published 23 March 2017

\begin{abstract}
There is a long standing contradiction on the tensile response of zinc oxide nanowires between theoretical prediction and experimental observations. Although it is proposed that there is a ductile behavior dominated by phase transformation, only an elastic deformation and brittle fracture was witnessed in experiments. Using molecular dynamics simulations, we clarified that, as the lateral dimension of zinc oxide nanowires increases to a critical value, an unambiguous ductile-to-brittle transition occurs. The critical value increases with decreasing the strain rate. Factors including planar defects and surface contamination induce brittle fracture prior to the initiation of phase transformation. These findings are consistent with previous atomistic standpoints and experimental results.
\end{abstract}

Keywords: $\mathrm{ZnO}$ nanowires, phase transformation, size effect, strain rate, molecular dynamics

(Some figures may appear in colour only in the online journal)

\section{Introduction}

Nanostructured zinc oxide $(\mathrm{ZnO})$ compounds are attractive materials due to their exceptional optical, electrical and mechanical properties. For these reasons, they are used in a wide range of applications including energy and environment [1-4], biomedical devices [5], nanoelectronics [6] and mechanical damping technology [7]. These applications mainly benefit from the unique surface feature of materials at the nanoscale where a large surface-to-volume ratio with a magnitude of $10^{6}-10^{9} \mathrm{~m}^{-1}$ breaks an atomic symmetry on the surface of nanostructured $\mathrm{ZnO}$ [8, 9]. Theoretical analysis based on molecular dynamics (MD) simulations have shown that there is noticeable surface stress, which increases Young's modulus of $\mathrm{ZnO}$ nanobelts as their lateral dimensions are reduced [10]. The finding was verified by subsequent experiments on $\mathrm{ZnO}$ nanowires (NWs) [11, 12]. The large surface-to-volume ratio also brings an atomic mobility with higher energy in $\mathrm{ZnO}$ nanostructures in contrast to their bulk counterparts. Thus, $\mathrm{ZnO}$ nanostructures are highly active to reconstruct under external stimulation. Phase transformation is such a reconstruction initiated by mechanical loading. The mechanically initiated hexagonal [13] and tetragonal [14] phases in $\mathrm{ZnO} \mathrm{NWs}$ can lead to continuous interests since similar transformations are prevalent in a large variety of IIVI, III-V and IV-IV wurtzite structured ceramics [15-18].

With the ability of phase transformation, nanoscale ceramics have two distinct advantages. First, phase transformation improves their deformability. Taking the wurtzite to tetragonal phase transformation in $\mathrm{ZnO} \mathrm{NWs}$ as an example, the initial wurtzite structured NWs yields $6.5 \%-10.0 \%$ strain at the elastic limit $[19,20]$. These values fall at the middle of experimentally measured fracture strain, $2 \%-15 \%$, of wurtzite structured ZnO NWs [19, 21-23]. After phase transformation, 
fracture strain reaches to $17 \%-20 \%[19,20]$. Most importantly, unloading of transformed NWs leaves $6 \%-7 \%$ residual strain, declaring a phase transformation dominated ductility [24]. Second, there is a reversible transition from wurtzite to tetragonal reconstruction that was observed on the $\{01 \overline{1} 0\}$ surface of $\mathrm{ZnO}$ nano-islands via in situ transmission electron microscopy [25]. This indicates that $\mathrm{ZnO}$ nanostructures have potential applications in shape memory devices [26].

Although the reversible wurtzite to tetragonal phase transformation offers $\mathrm{ZnO}$ nanostructures promising shape memory applications and the tetragonal phase has been experimentally witnessed [25, 27], there exists a puzzle between theoretical prediction and experimental observations. The tetragonal phase was firstly captured by MD in simulating the tensile deformation of $\mathrm{ZnO}$ NWs [14]. However, so far only brittle fracture of wurtzite structured $\mathrm{ZnO}$ NWs was observed in tensile experiments $[12,19]$. To resolve this conflict, a theoretical study was recently conducted based on MD simulations using the reactive force field and $a b$ initio calculations. It was found that the wurtzite to tetragonal phase transformation significantly depends on the lateral dimension of $\mathrm{ZnO} N W s$, strain rate and temperature [20]. Although the lateral dimension was up to $4.8 \mathrm{~nm}$ in simulations, it is still much smaller than the lowest dimension $(\sim 20 \mathrm{~nm})$ experimentally tested [12, 28]. Moreover, it is argued that the transformation was forbidden under temperatures below $600 \mathrm{~K}$ [20]. This view is against that of a previous large-scale density functional theory analysis, which shows that the transformation occurred at $0 \mathrm{~K}$ [29].

It is seen that the tensile response of $\mathrm{ZnO}$ NWs is still illusive with two unsolved problems. One is the necessary requirement permitting the wurtzite to tetragonal phase transformation. The other is a reasonable interpretation of the large dispersion in fracture strain, $2 \%-15 \%$, of wurtzite structured NWs [19, 21-23]. Motivated by these two contradictions, we performed systematic MD simulations on the tensile deformation of $\mathrm{ZnO} \mathrm{NWs}$ in this paper. In section 2, the simulation techniques are introduced. Section 3 is then dedicated to a detailed description of tensile deformation of $\mathrm{ZnO} \mathrm{NWs}$ with and without phase transformations. Factors that facilitate and hinder the phase transformation are discussed in section 4. Finally, some concluding remarks are given in section 5 .

\section{Simulation procedures}

\subsection{Microstructures in $\mathrm{ZnO} N W s$}

In $\mathrm{ZnO} \mathrm{NWs}$, three low-indexed growth directions, [0001], [2 $\overline{1} \overline{1} 0]$ and [2 $\overline{1} \overline{1} 3$, have been identified [30]. These NWs exhibit triangle, rectangle and hexagonal cross-sections. Here we focus on [0001]-orientated NWs with the hexagonal lateral shape because a tetragonal phase has been detected on these NWs and sufficient experimental data and theoretical data are available for comparison [12, 14, 19, 20]. Moreover, intrinsic point and planar defects as well as extrinsic factors such as surface contamination are prevalent in $\mathrm{ZnO}$ NWs
Table 1. Parameters of the interaction potentials for $\mathrm{ZnO}$ in equations (1) and (2) [33].

\begin{tabular}{llrcr}
\hline Atom pair & Type & $A(\mathrm{eV})$ & $\rho(\AA)$ & $C\left(\AA^{-1}\right)$ \\
\hline $\mathrm{Zn}-\mathrm{Zn}$ & Born-Mayer & 78.91 & 0.5177 & \\
$\mathrm{Zn}-\mathrm{O}$ & Born-Mayer & 257600 & 0.1396 & \\
$\mathrm{O}-\mathrm{O}$ & Morse & 0.1567 & 3.4050 & 1.164 \\
\hline
\end{tabular}

[7, 31, 32]. These factors can have undeniable impact on tensile behaviors of NWs. It has been confirmed that point defects cause the scatter of fracture strain of wurtzite structured NWs [21]. However, to the best of our knowledge, the role of planar defects and surface contamination in tension of $\mathrm{ZnO}$ NWs remains unexplored. Therefore, these two microstructures are also taken into account.

\section{2. $M D$ simulation}

The interatomic interactions in $\mathrm{ZnO}$ are described by an $a b$ initio based pair potential. Specifically, the non-coulombic interactions $V$ of short-range $\mathrm{Zn}-\mathrm{O}$ and $\mathrm{Zn}-\mathrm{Zn}$ are expressed by the Born-Mayer model, that is

$$
V\left(r_{i j}\right)=A \mathrm{e}^{-r_{i j} / \rho},
$$

where $r_{i j}$ is the distance between atoms $i$ and $j$, and $A$ and $\rho$ are parameters mainly related to the hardness and size of ionic species [33]. Moreover, a Morse-type potential,

$$
V\left(r_{i j}\right)=A\left[\left(1-\mathrm{e}^{-C\left(r_{i j}-\rho\right)}\right)^{2}-1\right],
$$

is employed to represent the short-range $\mathrm{O}-\mathrm{O}$ interaction, where $C$ is a fitting parameter and its value is listed in table 1 [33]. The long-range Coulombic interactions are considered by assigning cations and anions to an effective charge of $\pm 1.14 e$ (here $e$ is the unit of electronic charge).

As is known, surface contamination can stem from carbon during tensile tests in the chamber of a scanning electron microscope [12]. Platinum can also cause contamination when $\mathrm{ZnO}$ NWs are welded to the testing system by electron beam induced deposition [12]. Moreover, fabricating NWs by focused ion beam may introduce gallium ions [34]. Thus, it is reasonable to consider these effects as a realistic surface contamination scenario. However, to the best of our knowledge, there is still lack of such an interatomic potential between any of these three elements and $\mathrm{ZnO}$. In this work, to evaluate the effect of surface contamination on the tensile behavior of $\mathrm{ZnO} \mathrm{NWs}$, we resort to a recently developed Rahman-Stillinger-Lemberg potential that models the interaction between silver $(\mathrm{Ag})$ and particles in $\mathrm{ZnO}$, that is

$$
V\left(r_{i j}\right)=D \mathrm{e}^{y\left(1-\frac{r_{i j}}{r_{0}}\right)}+\sum_{k=1}^{3} \frac{a_{k}}{1+\mathrm{e}^{b_{k}\left(r_{i j}-c_{k}\right)}},
$$

where the related values of parameters $D, y, r_{0}, a_{k}, b_{k}$ and $c_{k}$ are listed in table 2 [35].

The motion of atoms was integrated using a Verlet leapfrog algorithm with a time step of $1 \mathrm{fs}$. The long-range Coulombic interactions were calculated by the Wolf sum [36]. To obtain free-standing configurations, samples were relaxed 
Table 2. Potential parameters for interaction between $\mathrm{Ag}$ and $\mathrm{ZnO}$ in equation (3) [35]. The units of $D$ and $a_{k}(k=1,2,3)$ are $\mathrm{eV} ; r_{0}$ and $c_{k}$ are $\AA ; b_{k}$ is $\AA^{-1}$; and $y$ is dimensionless.

\begin{tabular}{lrrr}
\hline Atom pair & \multicolumn{3}{c}{ Potential parameters } \\
\hline Ag-Zn & $D=185.4810$ & $r_{0}=1$ & $y_{1}=4.2084$ \\
& $a_{1}=-0.0231$ & $b_{1}=5.2619$ & $c_{1}=3.5369$ \\
& $a_{2}=-0.3764$ & $b_{2}=1.8000$ & $c_{2}=3.3262$ \\
& $a_{3}=0.0038$ & $b_{3}=0.9656$ & $c_{3}=0.6894$ \\
Ag-O & $D=136.2424$ & $r_{0}=1$ & $y_{1}=5.0865$ \\
& $a_{1}=-1.5273$ & $b_{1}=2.6489$ & $c_{1}=2.1128$ \\
& $a_{2}=1.8830$ & $b_{2}=2.6256$ & $c_{2}=1.6708$ \\
& $a_{3}=-0.3976$ & $b_{3}=1.0508$ & $c_{3}=0.4472$ \\
Ag-Ag & $D=206.4544$ & $r_{0}=1$ & $y_{1}=1.6171$ \\
& $a_{1}=-75.7945$ & $b_{1}=2.2129$ & $c_{1}=1.6720$ \\
& $a_{2}=-17.0348$ & $b_{2}=1.4495$ & $c_{2}=2.2826$ \\
& $a_{3}=-0.5642$ & $b_{3}=0.6884$ & $c_{3}=0.0914$ \\
\hline
\end{tabular}

for $200 \mathrm{ps}$ before stretching. To simulate tension and obtain the mechanical properties of $\mathrm{ZnO} \mathrm{NWs}$, an extra velocity was applied along the [0001] orientation at each integral step via a modified isothermal-isobaric ensemble [10, 37, 38]. This scheme stretched NWs with strain rates ranging from $2.5 \times 10^{9}$ to $0.25 \mathrm{~s}^{-1}$. The stress tensor was calculated by the classical virial formula and the system temperature was controlled by the Nosé-Hoover thermostat [39, 40]. All simulations were carried out by using a modified version of the DL_POLY package [38].

\subsection{Selection of the interatomic potential}

It is known that the accuracy and credibility of simulating results depend significantly on the interatomic potential employed. To choose a proper one, we have checked four types of potentials developed based on diverse physical models: a self-consistent potential with a Morse function [41], a Buckingham potential adopted a core-shell model [42], the reactive potential aiming to explore structures and reaction dynamics such as bond breaking and formation [43] and a new ab initio based potential for accurate simulation of phase transformation in $\mathrm{ZnO}$ [33]. The self-consistent one failed to predict the wurtzite to tetragonal phase transformation. The Buckingham type was non-reactive regarding to bond breaking and formation [20]. The reactive potential was also abandoned since it deviates from the prediction of the density functional theory at $0 \mathrm{~K}$. Finally, the $a b$ initio based potential was chosen because it performs well on the following four aspects: (i) the lattice constants of wurtzite and tetragonal phases it generated are consistent with that of the density functional theory $[14,33]$; (ii) the thermal properties reconstructed echo with the experimental results and thus the temperature effect on phase transformation is detectable $[33,44]$; (iii) surface energy was also modeled and this allows investigation on the size-dependent mechanical properties of ZnO NWs [33]; and (iv) most importantly, phonon dispersion calculated converges to the experimental data $[33,45]$. This feature offers precision to study the strain rate effect on deformation behaviors of NWs since the strain rate has much to do with the motion of phonons [46, 47].

\section{Simulation results}

\subsection{Wurtzite to tetragonal phase transformation}

A free standing $\mathrm{ZnO}$ NW adopts the wurtzite structure (i.e., the $P 6_{3} m c$ space group) as shown in figure 1(a), which was generated by the visual MD software [48]. After phase transformation, the space group changes to $P 4_{2} / \mathrm{mnm}$. The transformed phase was previously referred to as the bodycentered-tetragonal phase with four-atom rings, a term that has been widely applied to many II-VI, III-V and IV-IV compounds [14-18, 24]. Strictly speaking, however, since the four-atom ring at the center of a tetragonal lattice is perpendicular to those at the corners (see figure 1(a)), it is more precise to name the transformed phase as the tetragonal structure. The wurtzite to tetragonal transformation causes a three-stage stress-strain relation as a $\mathrm{ZnO} \mathrm{NW}$ is uniaxially stretched. Figure 1(b) shows that the first stage from point $\mathrm{A}$ to point $\mathrm{B}$ is the elastic stretching process of the original wurtzite structure. Then, a sudden stress drop from point $B$ to point $\mathrm{C}$ corresponds to a structural transformation. Finally, the transformed tetragonal structure is subjected to an elastic stretching from point $\mathrm{C}$ to point $\mathrm{D}$ before brittle fracture. Figure 1(c) illustrates that the stress drop initiated at point $\mathrm{B}$ is due to the breaking of $\mathrm{Zn}-\mathrm{O}$ bonds along the [0001] orientation. However, no fracture occurs due to formation of an equal number of $\mathrm{Zn}-\mathrm{O}$ bonds along the same direction next to the broken bonds. Breaking and formation of $\mathrm{Zn}-\mathrm{O}$ bonds repeat on alternate planes along the [0110] direction that transforms the original wurtzite NW to a tetragonal one [24].

\subsection{Size effect}

To focus on the size-dependent tensile behavior of $\mathrm{ZnO} \mathrm{NWs}$, a strain rate of $2.5 \times 10^{9} \mathrm{~s}^{-1}$ was chosen and temperature was maintained at $300 \mathrm{~K}$. To compare with available results, we started from a NW with a lateral dimension of $1.0 \mathrm{~nm}$. Figure 2(a) shows that the phase transformation initiates at the elastic limit with a strain of $8.0 \%$ and completes at the strain of $8.3 \%$. These values are consistent with those predicted by the Buckingham potential $[14,19]$. As the lateral dimension increases to $4.2 \mathrm{~nm}$, the critical strain to trigger phase transformation rebounds to $10.2 \%$ and the transformation finishes at $11.0 \%$ strain. This agrees with that obtained by the reactive force field [20]. However, this differs from an investigation using the Buckingham potential. The corresponding strains were $6.5 \%$ and $8.0 \%$, respectively, to trigger and complete phase transformation for a NW with a lateral dimension of $5 \mathrm{~nm}$ [19]. Taking bond rotation, bond breaking and bond formation into account, this difference is ascribed to failure of the Buckingham potential to capture the reactive feature in phase transformation [19]. Moreover, the previous investigation reveals that a transformed $\mathrm{NW}$ has a residual strain of $7 \%$ and maintains the tetragonal phase after unloading, 
(a)

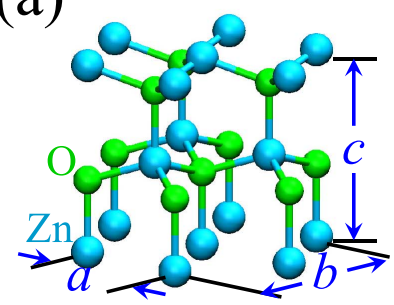

Wurtzite

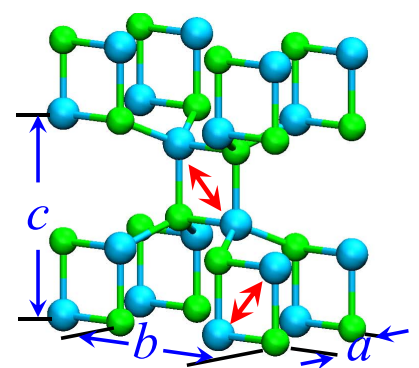

Tetragonal (b)

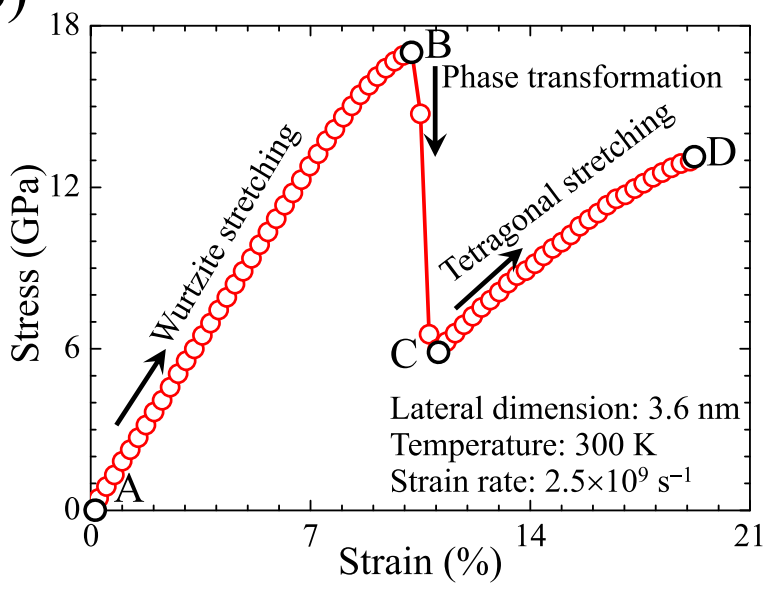

(c)

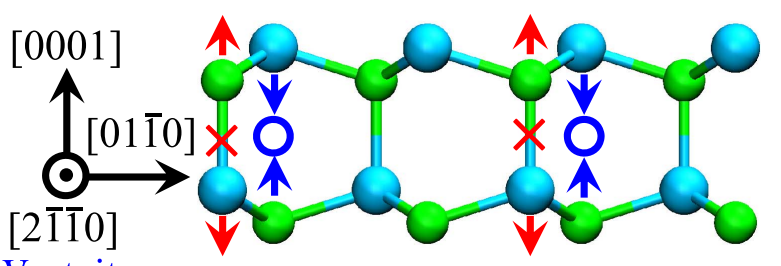

Wurtzite

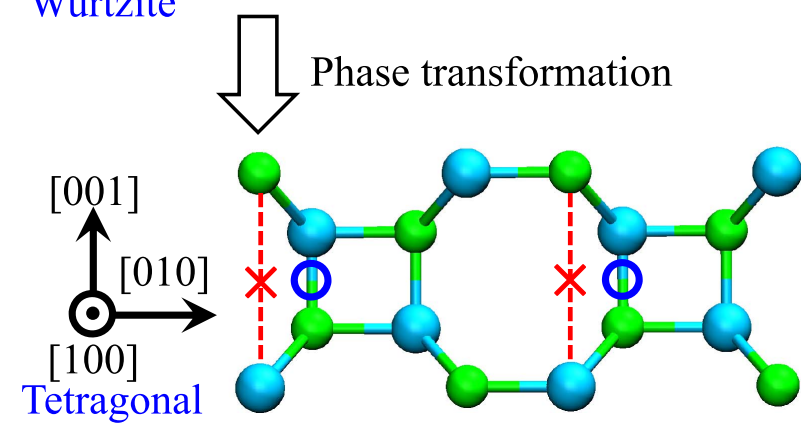

Figure 1. A sketch of the wurtzite-to-tetragonal phase transformation. (a) Wurtzite and tetragonal lattices. Two-way arrows point out mutually vertical orientations of four-atom rings located at the center and a corner of the tetragonal cell. (b) A typical stress-strain curve of $\mathrm{ZnO} \mathrm{NW}$ with tension along the [0001] orientation. (c) The path of phase transformation from wurtzite to tetragonal structure. Arrows show relative motion of atoms. Crosses $(x)$ and circles $(\bigcirc)$ mark positions where $\mathrm{Zn}-\mathrm{O}$ bonds break and form, respectively.

indicating a ductile behavior dominated by phase transformation [14, 24]. Here, it is worth noting that, however, an essential transition on the tensile behavior of $\mathrm{ZnO}$ NWs occurs as the lateral dimension exceeds $4.9 \mathrm{~nm}$. In this case, brittle fracture occurs as the wurtzite structured NW reaches its elastic limit with a strain of $10.2 \%$ and no phase (a)

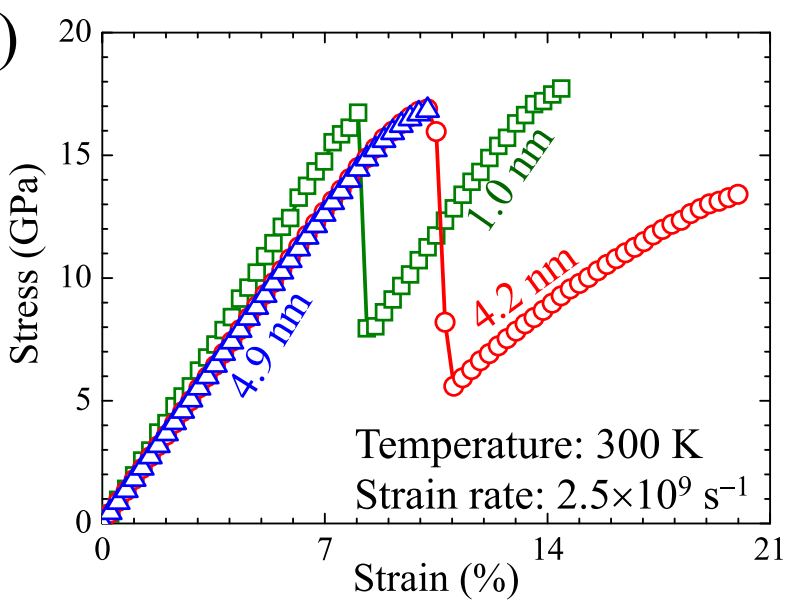

(b)

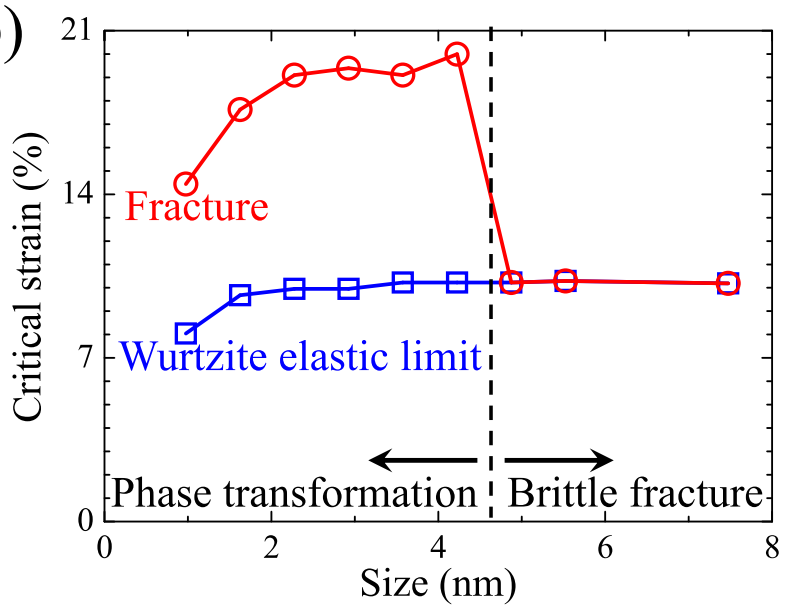

Figure 2. Size-dependent tensile behavior of $\mathrm{ZnO}$ NWs. (a) Stressstrain curves of $\mathrm{ZnO}$ NWs with various lateral dimensions. (b) Strain at the elastic limit of wurtzite structure and strain at brittle fracture with various lateral dimensions of NWs.

transformation is detected. This does not contradict with previous simulations where a sample with a lateral dimension of $5 \mathrm{~nm}$ showed phase transformation. The reason for this seeming contradiction is due to the fact that the NW underwent quasi-static deformation without considering the effect of strain rates [19].

Critical strains at the elastic limit of wurtzite and fracture altering with the lateral dimension of NWs are summarized in figure 2(b). It is shown that strain at the elastic limit of wurtzite gradually increases from $8.0 \%$ to $10.2 \%$ as the lateral dimension increases from 1.0 to $3.6 \mathrm{~nm}$. Then, the strain remains almost unchanged with the increase of lateral dimensions. Furthermore, fracture strain of $\mathrm{ZnO} \mathrm{NWs}$ increases from $14.4 \%$ to $20 \%$ as the lateral dimension increases from 1.0 to $4.2 \mathrm{~nm}$. However, it dramatically reduces to $10.2 \%$ with the lateral dimension beyond $4.9 \mathrm{~nm}$ due to elimination of phase transformation.

\subsection{Strain rate}

To describe the strain rate effect on tension of $\mathrm{ZnO} N W s$, a $\mathrm{NW}$ with a lateral dimension of $10.1 \mathrm{~nm}$ and an aspect ratio of 2:1 was employed. The simulation temperature was 


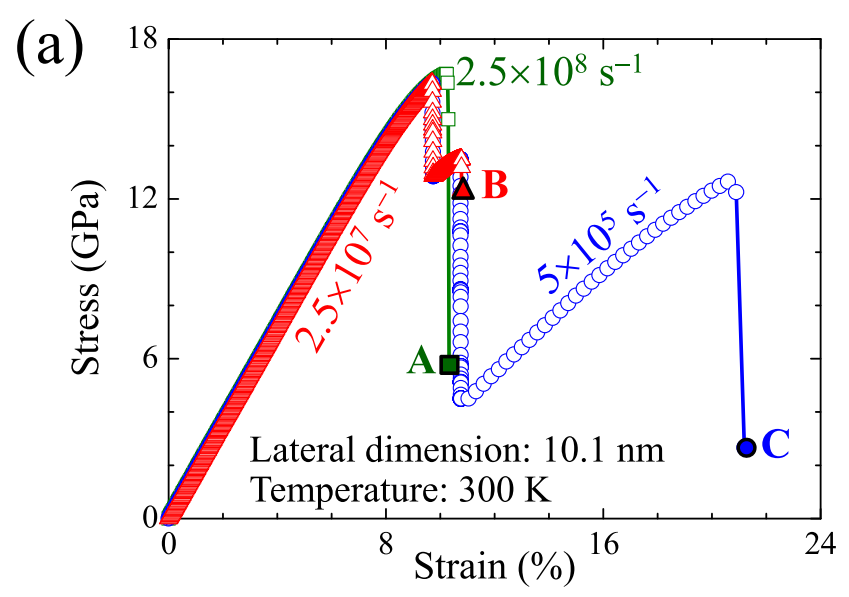

Point A

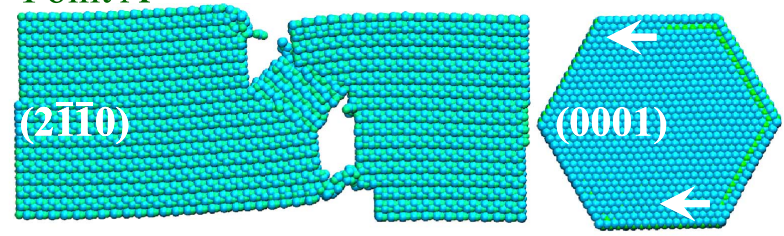

Point B

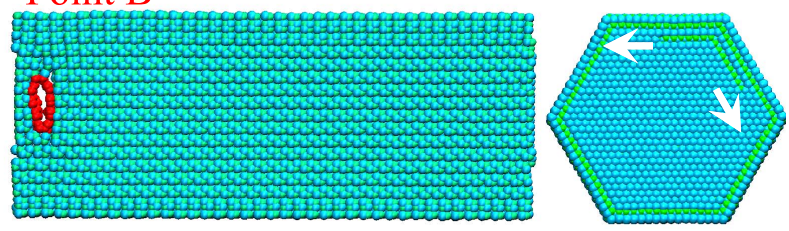

Point C

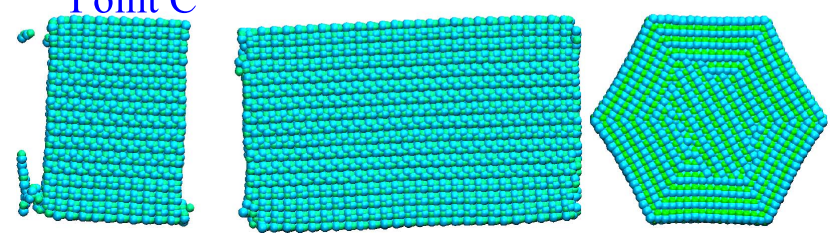

(b)

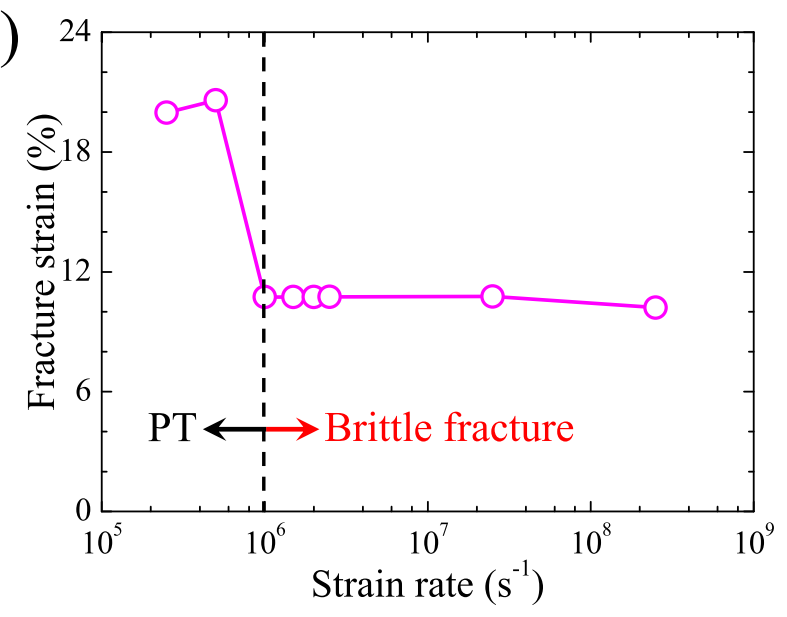

Figure 3. Effect of strain rate on tensile response of $\mathrm{ZnO}$ NWs. (a) Typical stress-strain curves with diverse strain rates. Arrows in snapshots represent spreading directions of tetragonal phase. Red atoms outline a crack. (b) Fracture strain varies with strain rate. PT indicates phase transformation. maintained at $300 \mathrm{~K}$. As shown in figure 3(a), at a strain rate of $2.5 \times 10^{8} \mathrm{~s}^{-1}$, the NW undergoes elastic stretching and the snapshot at point A reveals that phase transformation initiates at one ridge and propagates on its surface. However, propagation suddenly stops as soon as the NW exceeds its elastic limit and brittle fracture takes place. As the strain rate decreases to $2.5 \times 10^{7} \mathrm{~s}^{-1}$, the outmost surface layer of the NW completes transformation and the tetragonal phase starts to grow towards the NW core. Noticeably, the new phase still originates from the ridge side and spreads parallel to the NW surface until a crack perpendicular to the NW axis is generated at the intersection between wurtzite and tetragonal regions (see the snapshot at point B in figure 3(a)). The crack expands rapidly and causes brittle fracture, declaring the termination of phase transformation. To ensure transformation of the entire $\mathrm{NW}$, the strain rate has to be reduced to below $5 \times 10^{5} \mathrm{~s}^{-1}$, as shown by the snapshot at point $\mathrm{C}$ in figure $3(\mathrm{a})$. It is seen that fracture strain $(20.6 \%)$ of the transformed NW is at least 2 times of that $(10.2 \%)$ of the original wurtzite one.

Fracture strain of the NW versus strain rate is drawn in figure 3(b). The tensile response of the NW shows three kinds of attributes with the reduction of strain rates: (i) as the strain rate is beyond $2.5 \times 10^{7} \mathrm{~s}^{-1}$, brittle fracture of wurtzite structure dominates tensile deformation of the NW; (ii) with further decrease of strain rates, incomplete phase transformation takes over at the range between $2.5 \times 10^{7}$ and $1 \times 10^{6} \mathrm{~s}^{-1}$; and (iii) the completion of phase transformation is allowed with strain rates below $5 \times 10^{5} \mathrm{~s}^{-1}$.

It is seen in table 3 , the critical strain rate to initiate the wurtzite to tetragonal transformation reduces almost 10 orders of magnitude from $1 \times 10^{9}$ to $5 \times 10^{-1} \mathrm{~s}^{-1}$ as the lateral dimension of NWs increases from 4.9 to $19.8 \mathrm{~nm}$.

\subsection{Temperature effect}

To ascertain the temperature effect on transformation, a NW with a lateral dimension of $5.5 \mathrm{~nm}$ and an aspect ratio of 2:1 was examined. The applied strain rate was $2.5 \times 10^{9} \mathrm{~s}^{-1}$ and simulation temperature was from 300 to $600 \mathrm{~K}$. Figure 4(a) shows that as temperature is below $450 \mathrm{~K}$, only elastic deformation and brittle fracture of wurtzite structured NWs are observed. However, with temperature over this critical value, stress-strain curves represent the three-stage attribution, indicating occurrence and completion of phase transformation. Critical strains at various temperatures are presented in figure 4(b). It is seen that, due to thermal softening, strain at the elastic limit of the wurtzite structure decreases with the increase of temperature. This result is consistent with previous studies [24]. Most importantly, it is shown that $450 \mathrm{~K}$ is the critical temperature for the wurtzite to tetragonal phase transformation.

\subsection{Stacking faults}

The wurtzite structure is one of the close-packed crystals with a periodic $\mathrm{ABAB}$... stacking sequence. Here, $\mathrm{A}$ and $\mathrm{B}$ represent two of the three basic tetrahedral stacking units (i.e., 
Table 3. Critical strain rates to initiate phase transformation with various lateral dimensions of NWs at $300 \mathrm{~K}$.

\begin{tabular}{lcccccc}
\hline Strain rate $\left(\mathrm{s}^{-1}\right)$ & $2.5 \times 10^{9}$ & $1 \times 10^{9}$ & $4.5 \times 10^{8}$ & $1.2 \times 10^{8}$ & $5 \times 10^{5}$ & $5 \times 10^{-1}$ \\
Lateral dimension $(\mathrm{nm})$ & 4.2 & 4.9 & 5.5 & 7.5 & 10.1 & 19.8 \\
\hline
\end{tabular}

(a)

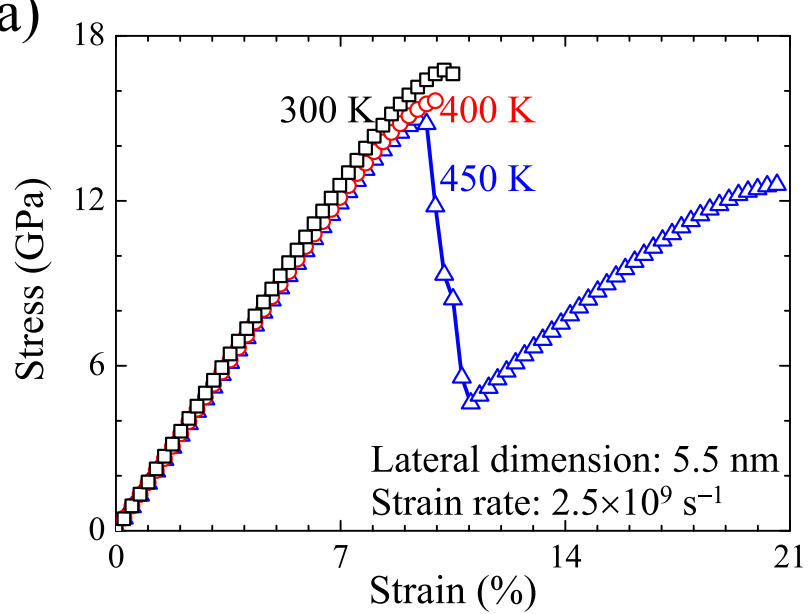

(b)

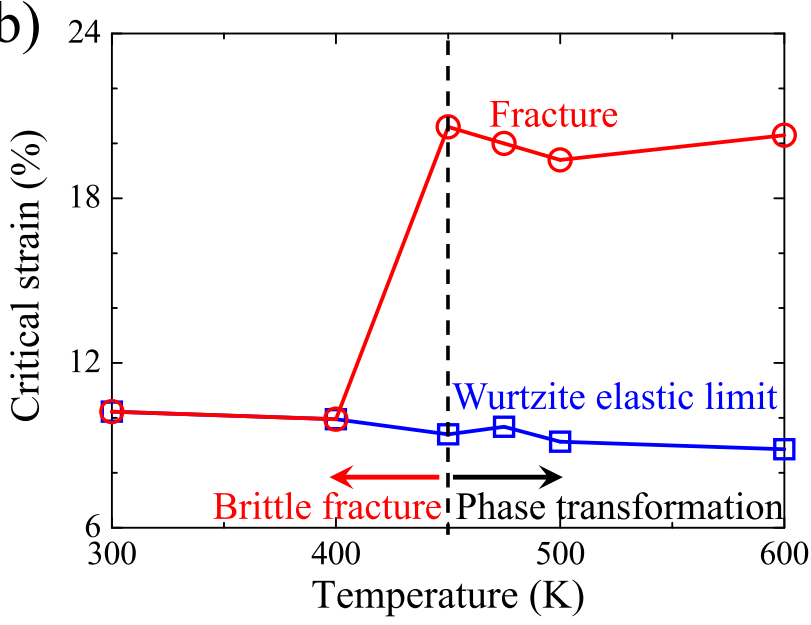

Figure 4. Temperature dependent deformation of $\mathrm{ZnO}$ NWs. (a) Stress-strain curves and (b) critical strains under several temperatures.

$\mathrm{A}, \mathrm{B}$ and $\mathrm{C}$ ) [49]. Inserting $\mathrm{C}$ into the $\mathrm{ABAB} \ldots$ sequence generates a stacking fault, see insets in figure 5(a). To investigate the effect of stacking faults on tensile deformation of $\mathrm{ZnO} \mathrm{NWs}$, a lateral dimension of $5.5 \mathrm{~nm}$ was considered. A strain rate of $4 \times 10^{8} \mathrm{~s}^{-1}$ was adopted and it is adequate to induce wurtzite to tetragonal transformation for a single crystal NW with the same lateral dimension at $300 \mathrm{~K}$. A stacking fault was introduced at the middle of a NW and the periodic boundary condition was used along the axis. In such a design, spacing between parallel-spaced stacking faults is from 10.9 to $552.8 \mathrm{~nm}$ and aspect ratios of NWs vary from $2: 1$ to $100: 1$. Results show that spacing has negligible impact on fracture strain, which is around $7.7 \%$ ranging from $7.6 \%$ to $7.9 \%$, see figure 5(a). Additionally, all samples maintained the original wurtzite structure until brittle fracture.

Figure 5(b) shows that $\mathrm{Zn}$ and $\mathrm{O}$ atoms at breaking $\mathrm{Zn}-\mathrm{O}$ bonds (sites marked by crosses) in a stacking fault fail to
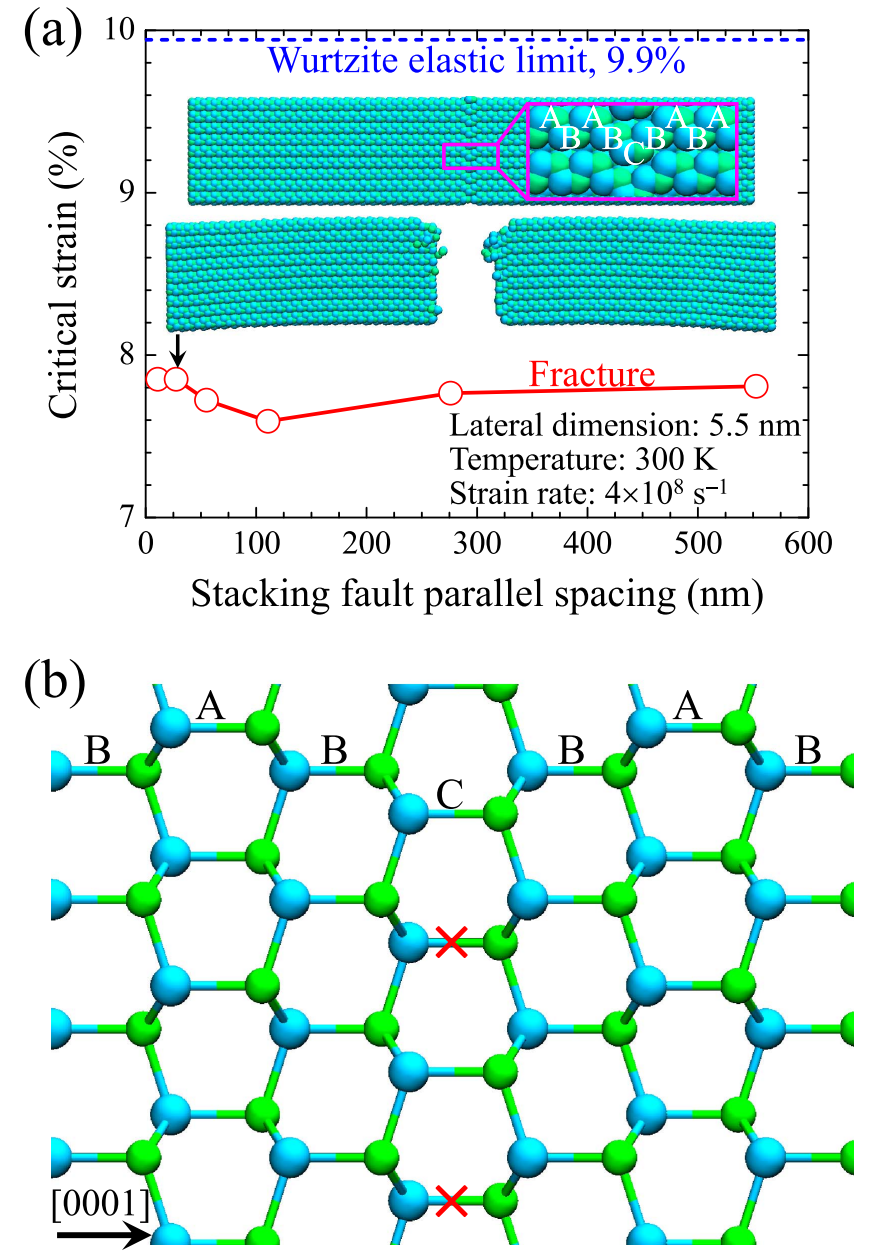

Figure 5. Stacking faults induce brittle fracture of wurtzite NWs. (a) Fracture strain of $\mathrm{ZnO}$ NWs with parallel spaced stacking faults. Insets show patterns of a NW with a parallel spacing of $27.6 \mathrm{~nm}$ between neighboring stacking faults before and after fracture. (b) Cracks initiate at sites marked by crosses $(\times)$ within a stacking fault.

reform along the [0001] orientation due to interruption of the wurtzite stacking regulation. As a result, cracks initiate at the breaking bonds and subsequently cause brittle fracture at the stacking fault.

\subsection{Surface contamination}

Contamination was considered by stochastically attaching Ag atoms on the surface of $\mathrm{ZnO}$ NWs. Ag atoms was introduced based on a concentration from 0.7 to 20 at $\%$. NWs involved had a lateral dimension of $5.5 \mathrm{~nm}$ and an aspect ratio of 2:1. Tensile deformation was conducted with a strain rate of $4 \times 10^{8} \mathrm{~s}^{-1}$ at $300 \mathrm{~K}$. These conditions ensure phase transformation of single crystalline wurtzite NWs with the same size. Results shown in figure 6 suggest that surface contamination hinders the wurtzite to tetragonal phase 


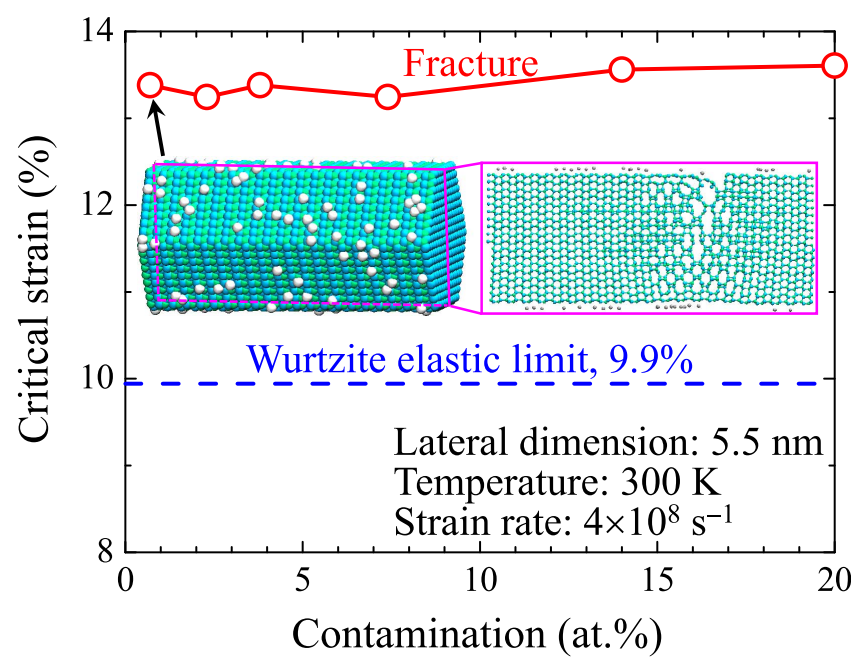

Figure 6. Fracture strain of $\mathrm{ZnO} \mathrm{NWs}$ contaminated with $\mathrm{Ag}$. Insets are original and fractured patterns of a NW with 0.7 at $\%$ surface contamination.

transformation. All samples are subjected to brittle fracture and the original wurtzite structure is maintained. However, it is of interest to note that even slight contamination could cause a substantial enhance of fracture strain. Taking the concentration of 0.7 at $\%$ for example, in comparison with that of the wurtzite structure, fracture strain was enhanced from 9.9\% to $13.4 \%$. Although Ag is not a realistic surface contaminant, the enhanced value is close to $12 \%-15 \%$ measured from experiments [22], where carbon, platinum and gallium ions were the most likely contaminants $[12,34]$.

\section{Discussion}

\subsection{Wurtzite to tetragonal phase transformation}

It is shown that wurtzite to tetragonal phase transformation in $\mathrm{ZnO} \mathrm{NWs}$ is significantly size, strain rate and temperature dependent. To reveal the underlying mechanisms, we resort to the Arrhenius equation, that is

$$
\dot{\varepsilon}_{\mathrm{t}}=B \mathrm{e}^{-E_{\mathrm{a}} / k_{\mathrm{B}} T},
$$

where $\dot{\varepsilon}_{\mathrm{t}}$ is the critical strain rate to trigger transformation, $B$ is the pre-exponential factor, $E_{\mathrm{a}}$ is the activation energy for the wurtzite to tetragonal transformation, and $k_{\mathrm{B}}$ and $T$ are the Boltzmann constant and temperature, respectively. Before applying equation (4) to predict the wurtzite to tetragonal transformation, it is noticed that the strain rate effect on mechanical behaviors results from the competition between two time scales. One is the external loading rate and the other is the frequency of thermal vibration of atoms in a material at a given temperature $[46,47]$. Here, we particularly claim that $\dot{\varepsilon}_{\mathrm{t}}$ and $B$ in equation (4) represent the two opponents. The former has been determined by simulations in this study. The latter is around $10^{13} \mathrm{~s}^{-1}$ according to the upper limit of optical phonons of $\mathrm{ZnO}$ [50]. In a recent work, the same value was assumed to deal with the wurtzite to tetragonal phase transformation in $\mathrm{ZnO}$ NWs [20]. After $B$ is ascertained, it is

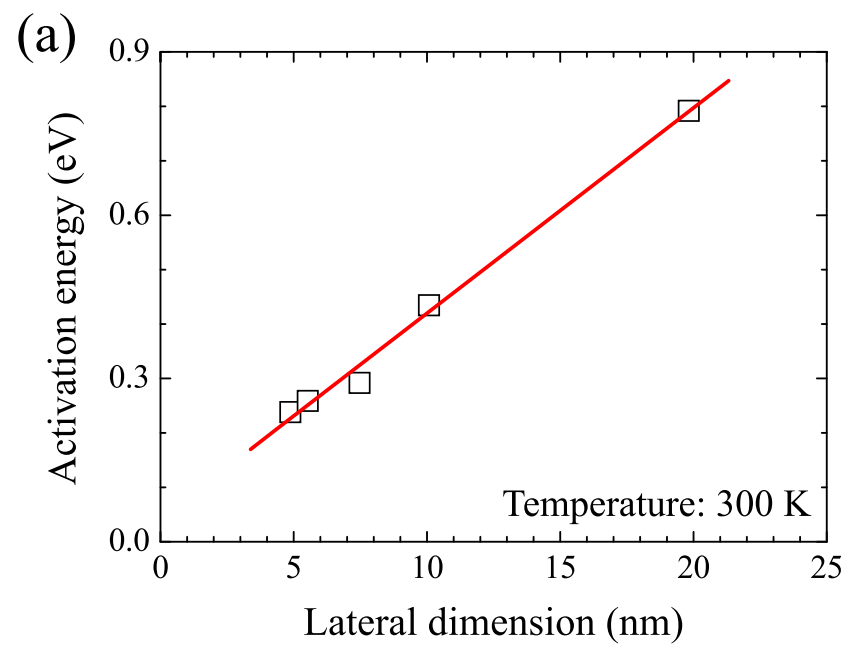

(b)

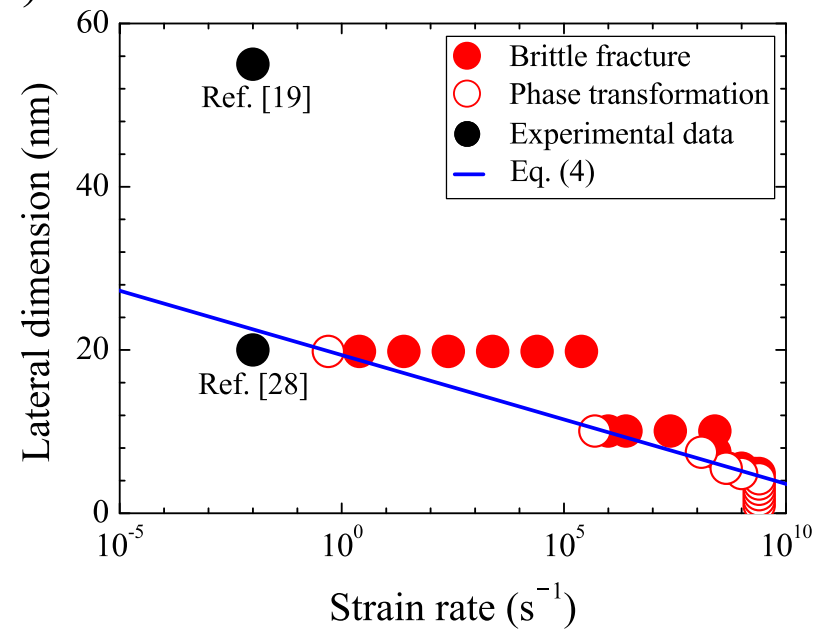

Figure 7. Size and strain rate dependent wurtzite-to-tetragonal phase transformation in $\mathrm{ZnO}$ NWs. (a) The activation energy grows proportionally as the lateral dimension of $\mathrm{ZnO}$ NWs increases. The line is a visual guide for the trend. (b) A summary of deformation behaviors of $\mathrm{ZnO} \mathrm{NWs}$ with various lateral dimensions and strain rates. The line represents critical values below which phase transformation is allowed.

shown that the activation energy increases with the increase of the lateral dimension of NWs (see figure 7(a)). This is not surprising since the increase of lateral dimension cuts down the cohesive energy of a material due to reduction of surface region [26]. Thus, the energy barrier between wurtzite and tetragonal structures grows as the lateral dimension of NWs increases.

To compare the prediction of equation (4) with experimental results, let us review tensile protocols in the microelectro-mechanical system based on the nanoscale testing technique $[12,19,28]$. According to this technique, an axial displacement is generated using a thermal actuator and loads are measured using a differential capacitive-based load sensor $[34,51]$. The displacement-capacitance change relationship is calibrated by actuating an identical device with a series of stepwise increasing voltages [34]. The voltages are applied sequentially in several on-off cycles with each cycle lasting $20 \mathrm{~s}$ [34]. Here, we assume the strain rate is $10^{-2} \mathrm{~s}^{-1}$ given 
that in situ tensile experiments were conducted under the quasi-static condition. By summarizing simulation results, experimental outcomes and prediction of equation (4) (see figure 7(b)), we find out that the case of $55 \mathrm{~nm}$ in [19] agrees well with the prediction of equation (4). However, tensile behavior of the $20 \mathrm{~nm}$ sample conflicts with prediction since the wurtzite to tetragonal phase transformation was not observed [28]. The absence of transformation could be caused by defects such as stacking faults and surface contamination, as indicated in sections 3.5 and 3.6.

\subsection{Large dispersion of fracture strain of wurtzite $\mathrm{ZnO} N W \mathrm{~s}$}

Our study reveals that fracture strain of $\mathrm{ZnO}$ NWs depends on the wurtzite to tetragonal phase transformation. Fracture strains generated by wurtzite and tetragonal structures are around $10 \%$ and $20 \%$, respectively. Here, we focus on the former by considering available experimental data of wurtzite structure. Based on our investigation, existence of defects leads to the large dispersion of fracture strain (hereafter refer to that of wurtzite NWs). Qualitatively different defects such as stacking faults and surface contamination could either weaken or enhance fracture strain.

Stacking faults are kind of defects which reduce fracture strain. As shown in figure 5, stacking faults yield fracture strains around $7.7 \%$ which are smaller than that, $9.9 \%$, of a single crystalline wurtzite $\mathrm{NW}$ with a lateral dimension of $5.5 \mathrm{~nm}$. The stacking fault generated fracture strain remains almost unchanged even as the parallel spacing between two adjacent stacking faults is up to $553 \mathrm{~nm}$, which is equivalent to a dense of planar defect of $4.7 \times 10^{-4}$ at $\%$. A stacking fault breaks a periodic stacking regulation of wurtzite sequence and causes the lattice disregistry in its neighboring layers, leading to a natural source of cracks near these layers. A similar role of stacking faults caused weakening of fracture strain was confirmed in silicon carbide NWs [52]. Moreover, point defects such as vacancies are also reasons for resulting in reduction of fracture strain. The MD simulations have proven that vacancies can lower strain at the elastic limit of wurtzite $\mathrm{ZnO} \mathrm{NWs}$ to $4 \%$. This value falls in the middle of experimental tests, $2 \%-7 \%$ [21].

On the contrary, surface contamination is the reason to raise fracture strain. Nanomaterials are known to have considerable dangling bonds at their surface area due to the large surface-to-volume ratio [8,9]. These bonds are highly active to react mutually or with an introducing impurity such as surface contamination. This inclination is utilized to improve deformability of a material and delay initiation of cracks at the surface area. This approach is referred to as the surface modification. Thus, facture strain of a NW reaches to $13.4 \%$ with the introduction of 0.7 at $\%$ surface impurity, as depicted in figure 6. The enhanced fracture strain, $13.4 \%$, is well consistent with the upper bound, $12 \%-15 \%$, experimentally discovered in wurtzite $\mathrm{ZnO}$ NWs [22]. Surface modification is also implemented to enlarge fracture strain of a lot of materials $[53,54]$.

\section{Conclusions}

We have shown that there is a wurtzite to tetragonal phase transformation in tensile behaviors of [0001]-oriented wurtzite $\mathrm{ZnO}$ NWs by means of MD simulations. Our systematic calculation reveals that the transformation depends significantly on the NW size and strain rate. By adopting the Arrhenius equation, these effects are elucidated through the competition between internally thermal motion of atoms and external loading rate. The former benefits the transformation whilst the latter dominates brittle fracture. Further, the large dispersion of fracture strain experimentally observed is attributed to the influence of defects. On one hand, stacking faults weaken fracture strain due to the lattice disregistry. On the other, surface contamination reinforces fracture strain via modification of dangling bonds on the surface of a NW. The finding provides a better understanding of how the wurtzite to tetragonal phase transformation occurs in a lot of II-VI, III-V and IV-IV ceramics. It has also highlighted the potential of tuning the mechanical behavior of nanostructures with defect engineering.

\section{Acknowledgments}

This work was supported by the National Natural Science Foundation of China (Grant Nos. 11372022 and 11232013), the Opening Fund of State Key Laboratory of Nonlinear Mechanics, the Strategic Priority Research Program of the Chinese Academy of Sciences (Project No. XDB22040501) and the Research Grant Council of the Hong Kong Special Administrative Region, China (Project No. 9042201 (CityU 11211015)). Computations were performed on the resources provided by the Pawsey Supercomputing Center with funding from the Australian Government and the Government of Western Australia, the LNMGrid of the State Key Laboratory of Nonlinear Mechanics and the ScGrid of Supercomputing Center, Computer Network Information Center of the Chinese Academy of Sciences.

\section{References}

[1] Wang Z L and Song J H 2006 Piezoelectric nanogenerators based on zinc oxide nanowire arrays Science 312 242-6

[2] Schrauben J H, Hayoun R, Valdez C N, Braten M, Fridley L and Mayer J M 2012 Titanium and zinc oxide nanoparticles are proton-coupled electron transfer agents Science 336 1298-301

[3] You J et al 2016 Improved air stability of perovskite solar cells via solution-processed metal oxide transport layers $\mathrm{Nat}$. Nanotechnol. 11 75-81

[4] van Lare C, Lenzmann F, Verchuuren M A and Polman A 2015 Dielectric scattering patterns for efficient light trapping in thin-film solar cells Nano Lett. 15 4846-52

[5] Wahab R, Kaushik N, Khan F, Kaushik N K, Choi E H, Musarrat J and Al-Khedhairy A A 2016 Self-styled ZnO nanostructures promotes the cancer cell damage and supresses the epithelial phenotype of glioblastoma Sci. Rep. 619950 
[6] Fang L, Liu B, Liu L, Li Y, Huang K and Zhang Q 2016 Direct electrochemistry of glucose oxidase immobilized on $\mathrm{Au}$ nanoparticles-functionalized 3D hierarchically $\mathrm{ZnO}$ nanostructures and its application to bioelectrochemical glucose sensor Sensors Actuators B 222 1096-102

[7] Cheng G, Miao C, Qin Q, Li J, Xu F, Haftbaradaran H, Dickey E C, Gao H and Zhu Y 2015 Large anelasticity and associated energy dissipation in single-crystalline nanowires Nat. Nanotechnol. 10 687-91

[8] Ma F and Xu K 2006 Surface induced electron redistribution: a mechanism for mechanical strengthening of Au nanowires Scr. Mater. 55 951-4

[9] Liang W and Zhou M 2005 Pseudoelasticity of single crystalline $\mathrm{Cu}$ nanowires through reversible lattice reorientations Trans. ASME, J. Eng. Mater. Technol. 127 423-33

[10] Kulkarni A J, Zhou M and Ke F J 2005 Orientation and size dependence of the elastic properties of zinc oxide nanobelts Nanotechnology 16 2749-56

[11] Chen C Q, Shi Y, Zhang Y S, Zhu J and Yan Y J 2006 Size dependence of Young's modulus in $\mathrm{ZnO}$ nanowires Phys. Rev. Lett. 96075505

[12] Agrawal R, Peng B, Gdoutos E E and Espinosa H D 2008 Elasticity size effects in $\mathrm{ZnO}$ nanowires-a combined experimental-computational approach Nano Lett. 8 3668-74

[13] Kulkarni A J, Zhou M, Sarasamak K and Limpijumnong S 2006 Novel phase transformation in $\mathrm{ZnO}$ nanowires under tensile loading Phys. Rev. Lett. 97105502

[14] Wang J, Kulkarni A J, Sarasamak K, Limpijumnong S, Ke F J and Zhou M 2007 Molecular dynamics and density functional studies of a body-centered-tetragonal polymorph Of ZnO Phys. Rev. B 76172103

[15] Fu B, Chen L, Xie Y, Feng J and Ye X 2015 Novel mechanical behaviors of wurtzite CdSe nanowires J. Nanopart. Res. 17354

[16] Wen B, Zhao J, Melnik R and Tian Y 2011 Body-centered tetragonal $\mathrm{B}_{2} \mathrm{~N}_{2}$ : a novel sp3 bonding boron nitride polymorph Phys. Chem. Chem. Phys. 13 14565-70

[17] Jung K, Cho M and Zhou M 2014 Structure and thermomechanical behavior of bent $\mathrm{GaN}$ nanowires Comp. Mater. Sci. 81 524-9

[18] Fujimoto Y, Koretsune T, Saito S, Miyake T and Oshiyama A 2008 A new crystalline phase of four-fold coordinated silicon and germanium New J. Phys. 10083001

[19] Agrawal R, Peng B and Espinosa H D 2009 Experimentalcomputational investigation of $\mathrm{ZnO}$ nanowires strength and fracture Nano Lett. 9 4177-83

[20] Soldano G J, Zanotto F M and Mariscal M M 2015 Mechanical stability of zinc oxide nanowires under tensile loading: is wurtzite stable at the nanoscale? RSC Adv. 5 43563-70

[21] He M R, Xiao P, Zhao J, Dai S, Ke F J and Zhu J 2011 Quantifying the defect-dominated size effect of fracture strain in single crystalline $\mathrm{ZnO}$ nanowires J. Appl. Phys. 109 123504

[22] Desai A V and Haque M A 2007 Mechanical properties of $\mathrm{ZnO}$ nanowires Sensors Actuators A 134 169-76

[23] Hoffmann S, Östlund F, Michler J, Fan H J, Zacharias M, Christiansen S H and Ballif C 2007 Fracture strength and Young's modulus of $\mathrm{ZnO}$ nanowires Nanotechnology 18 205503

[24] Wang J, Kulkarni A J, Ke F J, Bai Y L and Zhou M 2008 Novel mechanical behavior of $\mathrm{ZnO}$ nanorods Comput. Methods Appl. Mech. Eng. 197 3182-9

[25] He M R, Yu R and Zhu J 2012 Reversible wurtzite-tetragonal reconstruction in $\mathrm{ZnO}(100)$ surfaces Angew. Chem., Int. Ed. $517744-7$

[26] Ma F, Xu K W and Chu P K 2013 Surface-induced structural transformation in nanowires Mater. Sci. Eng. R 74 173-209
[27] Wang F et al 2016 Nanometre-thick single-crystalline nanosheets grown at the water-air interface Nat. Commun. 7 10444

[28] Xu F, Qin Q, Mishra A, Gu Y and Zhu Y 2010 Mechanical properties of $\mathrm{ZnO}$ nanowires under different loading modes Nano Res. 3 271-80

[29] Agrawal R, Paci J T and Espinosa H D 2010 Large-scale density functional theory investigation of failure modes in $\mathrm{ZnO}$ nanowires Nano Lett. 10 3432-8

[30] Ding Y, Zhang F and Wang Z L 2013 Deriving the threedimensional structure of $\mathrm{ZnO}$ nanowires/nanobelts by scanning transmission electron microscope tomography Nano Res. 6 253-62

[31] Ding Y and Wang Z L 2009 Structures of planar defects in $\mathrm{ZnO}$ nanobelts and nanowires Micron 40 335-42

[32] Orsini A, Medaglia P G, Scarpellini D, Pizzoferrato R and Falconi C 2013 Towards high-performance, low-cost quartz sensors with high-density, well-separated, vertically aligned $\mathrm{ZnO}$ nanowires by low-temperature, seed-less, single-step, double-sided growth Nanotechnology 24355503

[33] Wang S, Fan Z, Koster R S, Fang C, van Huis M A, Yalcin A O, Tichelaar F D, Zandbergen $\mathrm{H}$ W and Vlugt T J H 2014 New ab initio based pair potential for accurate simulation of phase transitions in $\mathrm{ZnO} J$. Phys. Chem. C 118 11050-61

[34] Espinosa H D, Zhu Y and Moldovan N 2007 Design and operation of a MEMS-based material testing system for nanomechanical characterization J. Microelectromech. Syst. 16 1219-31

[35] Song H Q, Shen J, Qian P and Chen N X 2014 Interfacial potential approach for $\mathrm{Ag} / \mathrm{ZnO}$ (0001) interfaces Chin. Phys. B 23126801

[36] Wolf D, Keblinski P, Phillpot S R and Eggebrecht J 1999 Exact method for the simulation of Coulombic systems by spherically truncated, pairwise $\mathrm{r}-1$ summation $J$. Chem. Phys. 110 8254-82

[37] Melchionna S, Ciccotti G and Holian B L 1993 Hoover NPT dynamics for systems varying in shape and size Mol. Phys. 78 533-44

[38] Smith W, Yong C W and Rodger P M 2002 DL_POLY: applications to molecular simulation Mol. Simul. 28 385-471

[39] Nosé S 1984 A molecular dynamics method for simulations in the canonical ensemble Mol. Phys. 52 255-68

[40] Hoover W G 1985 Canonical dynamics: equilibrium phasespace distribution Phys. Rev. A 31 1695-7

[41] Binks D J and Grimes R W 1993 Incorporation of monovalent ions in $\mathrm{ZnO}$ and their influence on varistor degradation J. Am. Ceram. Soc. 76 2370-2

[42] Pedone A, Malavasi G, Menziani M C, Cormack A N and Segre U 2006 A new self-consistent empirical interatomic potential model for oxides, silicates, and silica-based glasses J. Phys. Chem. B 110 11780-95

[43] Raymand D, Duin A V, Baudin M and Hermansson K 2008 A reactive force field (ReaxFF) for zinc oxide Surf. Sci. $\mathbf{6 0 2}$ 1020-31

[44] Iwanaga H, Kunishige A and Takeuchi S 2000 Anisotropic thermal expansion in wurtzite-type crystals J. Mater. Sci. 35 2451-4

[45] Serrano J, Manjón F J, Romero A H, Ivanov A, Cardona M, Lauck R, Bosak A and Krisch M 2010 Phonon dispersion relations of zinc oxide: inelastic neutron scattering and ab initio calculations Phys. Rev. B 81174304

[46] Xiao P, Wang J, Yang R, Ke F J and Bai Y L 2015 Transition of mechanisms underlying the rate effects and its significance Comput. Mater. Sci. 98 70-5

[47] Xiao P, Wang J, Yang R, Ke F J, Xia M F and Bai Y L 2013 Rate effect and coupled evolution of atomic motions and potential landscapes Acta Mech. Sin. 29 676-81 
[48] Humphrey W, Dalke A and Schulten K 1996 VMD: visual molecular dynamics J. Mol. Graph. 14 33-8

[49] Lindefelt U, Iwata H, Öberg S and Briddon P R 2003 Stacking faults in $3 \mathrm{C}-, 4 \mathrm{H}-$, and $6 \mathrm{H}-\mathrm{SiC}$ polytypes investigated by an ab initio supercell method Phys. Rev. B 67155204

[50] Wang Z, Wang F, Wang L, Jia Y and Sun Q 2013 Firstprinciples study of negative thermal expansion in zinc oxide J. Appl. Phys. 114063508

[51] Wang W, Li S, Zhang H and Lu Y 2015 Recent developments in testing techniques for elastic mechanical properties of 1D nanomaterials Recent Patents Nanotechnol. 9 33-42
[52] Cheng G, Chang T H, Qin Q, Huang H and Zhu Y 2014 Mechanical properties of silicon carbide nanowires: effect of size-dependent defect density Nano Lett. 14 754-8

[53] Zhang L J, Yang D L, Qiu F, Wang J G and Jiang Q C 2015 Effects of reinforcement surface modification on the microstructures and tensile properties of $\mathrm{SiCp} / \mathrm{Al} 2014$ composites Mater. Sci. Eng. A 624 102-9

[54] Shikinaka K, Abe A and Shigehara K 2015 Nanohybrid film consisted of hydrophobized imogolite and various aliphatic polyesters Polymer 68 279-83 